\title{
The roles of Asp and Patronin in mitotic spindle formation in Drosophila
}

\author{
A. Razuvaeva ${ }^{1,2 *}$, G. Pavlova ${ }^{1}$, J. Popova ${ }^{1,3}$, L. Yarinich $^{1,2}$, M. Lebedev ${ }^{1,2}$, \\ E. Andreyeva ${ }^{1}$, A. Anders ${ }^{1,2}$, M. Gatti ${ }^{4}$, A. Pindyurin ${ }^{1,2,3}$ \\ ${ }^{1}$ Institute of Molecular and Cellular Biology SB RAS, Novosibirsk, Russia \\ ${ }^{2}$ Novosibirsk State University, Novosibirsk, Russia \\ ${ }^{3}$ Institute of Cytology and Genetics SB RAS, Novosibirsk, Russia \\ ${ }^{4}$ Sapienza University of Rome, Rome, Italy \\ *e-mail: alena.razuvaeva@mcb.nsc.ru
}

Key words: microtubule, mitotic spindle, kinetochore, colcemid, Drosophila

Motivation and Aim: Proper formation of a functional mitotic spindle in centrosomecontaining cells requires the concerted action of several microtubule- (MT-) biding proteins. They include proteins that bind the MTs lateral walls (e. g., Dgt6, a subunit of the augmin complex), MT plus end-associated proteins and MT minus end-binding proteins (e.g., Asp and Patronin). While the roles of the plus end-binding proteins in spindle assembly are rather well known, the precise functions of the minus end-binding factors are still poorly defined. Here, we analyzed the relationships between Asp, Dgt6 and Patronin in Drosophila spindle assembly and our results suggest a model on how these proteins cooperate to ensure proper spindle formation and functioning.

Methods and Algorithms: To understand the roles of Asp, Patronin and Dgt6, we performed RNAi in Drosophila S2 cells against the genes that encode these proteins and examined the ensuing mitotic phenotypes. To define the functional relationships among these genes, we also performed double RNAi against gene pairs. In addition, we generated S2 cell lines that stably express Asp-eGFP or Patronin-eGFP fusion proteins. For Dgt6 localization we used a previously generated antibody that specifically reacts with Dgt6.

Results: Immunostaining experiments revealed that Patronin-eGFP preferentially associates with the kinetochore fibers during mitosis showing a localization pattern rather similar to that of Dgt6. In contrast, Asp-eGFP accumulates at the minus ends of the spindle pole MTs, consistent with previous results. Moreover, results of RNAi experiments together with localization of eGFP fusion proteins suggest that Asp and Patronin functions during mitosis are at least in part independent. Double RNAitreatment for asp and Patronin genes resulted in a much stronger phenotype than that observed after RNAi against each of the genes. In double asp and Patronin RNAi cells we observed failure of sister chromatid separation and metaphase arrest.

Conclusion: Our findings suggest that Asp and Patronin are involved in different pathways required for spindle formation. They also raise the possibility that Patronin specifically binds and stabilize the minus ends of MTs that grow from the augmin-coated lateral walls of preexisting MTs. However these conclusions are the moment only working hypotheses, as they require additional experimental support.

Acknowledgements: Supported by the RFBR (18-34-00688). 